\title{
$\mathrm{Sn}$ 도금을 통한 $\mathrm{PCB}$ 기판상의 솔더 범프 제작 및 미세조직 분석 김상혁 ${ }^{1}$ · 김성진 ${ }^{1} \cdot$ 신한균 ${ }^{1} \cdot$ 박 현 $^{1} \cdot$ 허철호 $^{2} \cdot$ 문성재 ${ }^{2} \cdot$ 이효종 ${ }^{1, *}$ \\ 1동아대학교 공과대학 신소재공학과 2삼성전기 기판사업부
}

\section{Microstructural Analysis of Solder Bump Fabricated by Sn Electroplating on a PCB Substrate}

\author{
Sang-Hyeok Kim ${ }^{1}$, Seong-Jin Kim ${ }^{1}$, Han-Kyun Shin ${ }^{1}$, Hyun Park ${ }^{1}$, Cheol-Ho Heo ${ }^{2}$, \\ Seongjae Moon $^{2}$, and Hyo-Jong Lee ${ }^{1, *}$ \\ ${ }^{1}$ Department of Materials Science and Engineering, Dong-A University, Busan 49315, Republic of Korea \\ ${ }^{2}$ Substrate Solution, Samsung Electro-Mechanics, Busan 46754, Republic of Korea
}

\begin{abstract}
To manufacture finer solder bumps, the SR and DFR patterns were filled using a Sn electroplating process instead of the microball process currently used in BGA technology, and the solder bump shape was fabricated through a reflow process. The microstructure of the solder bump was investigated by EBSD and TEM measurements. The EBSD results showed that the grain size of the Sn structure became finer after the reflow treatment and a scallop shape of $\mathrm{Cu}_{6} \mathrm{Sn}_{5}$ was formed on the $\mathrm{Cu}$ UBM. However, the $\mathrm{Cu}_{3} \mathrm{Sn}$ phase was difficult to measure in the EBSD measurement. The $\mathrm{Cu}_{3} \mathrm{Sn}$ compound could be investigated with TEM analysis. The $\mathrm{Cu}_{3} \mathrm{Sn}$ phase also existed in the $\mathrm{Sn}$ region, with a size of several tens of nanometers, due to the eutectic reaction. The volume fraction of the $\mathrm{Cu}_{6} \mathrm{Sn}_{5}$ phase in the $\mathrm{Sn}$ region could be calculated from the TEM image, and the concentration of copper dissolved in the liquid tin during the reflow process could be estimated from the volume fraction. It was possible to observe the $\mathrm{Cu}_{3} \mathrm{Sn}$ and $\mathrm{Cu}_{6} \mathrm{Sn}_{5}$ lattice images through high resolution TEM analysis, but it was difficult to observe the lattice coherency between the two phases because both were polycrystalline. Based on the results of this study, it is expected that solder bumps with a diameter of less than $100 \mu \mathrm{m}$ can be robustly manufactured through the Sn electroplating process.
\end{abstract}

(Received December 2, 2020; Accepted February 8, 2021)

Keywords: electroplating, wetting, soldering, electron backscattering diffraction (EBSD)

\section{1. 서 론}

반도체 소자의 발전은 단위면적당 트렌지스터의 수로 표 현되는 무어의 법칙을 따라 발전을 거듭하고 있으며, 전자 부품의 실장을 위한 솔더 범프 피치(solder bump pitch)의 미세화도 전자기기의 발전과 더불어 지속되고 있다 [1]. 반 도체 웨이퍼에서는 이미 TSV(through silicon via) 및 $\mathrm{Cu}$ pillar 솔더 범프(solder bump) 공정기술이 적용되어 3차원 적층 및 선폭 미세화에 대응하고 있으며, 이러한 $\mathrm{Si}$ 칩

- 김상혁·신한균: 박사과정, 김성진: 석사과정, 박현·허철호·문성재: 연구원, 이효종: 교수

*Corresponding Author: Hyo-Jong Lee

[Tel: +82-51-200-7747, E-mail: hyojong@dau.ac.kr]

Copyright (c) The Korean Institute of Metals and Materials (chip)은 기판과 연결하는 $\mathrm{BGA}$ (ball grid array) 기술을 통해 전자부품에 활용되고 있다 [2]. 통상 $\mathrm{BGA}$ 기술은 마이크로 볼(microball)을 적절한 위치에 배치시켜 접합하 는 기술로 $100 \mu \mathrm{m}$ 이하의 ball size에 대한 공정 대응에 어려움이 있다. 본 연구는 향후 미세화될 범프 피치에 효 과적으로 대응할 수 있도록 도금공정을 이용하여 $\mathrm{Sn}$ 솔더 범프를 제작하고, 도금 직후 및 리플로우 공정 후의 미세 조직 변화를 $\mathrm{EBSD}$ (electron backscattered diffraction) 및 $\mathrm{TEM}$ (transmission electron microscopy)을 통해 분석한 결과를 담고 있다. 특히 $\mathrm{EBSD}$ 측정을 통한 솔더층의 결 정학적 미세조직은 $\mathrm{EM}$ (electromigration) 신뢰성과 관련되 어 있으며, $\mathrm{Sn}$ 결정립의 c축과 평행하거나 결정립이 조대 한 경우에 $\mathrm{EM}$ 결함이 나타나는 것으로 알려져 있다. 또한 


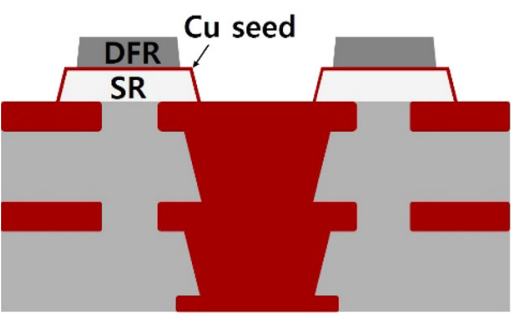

(a)

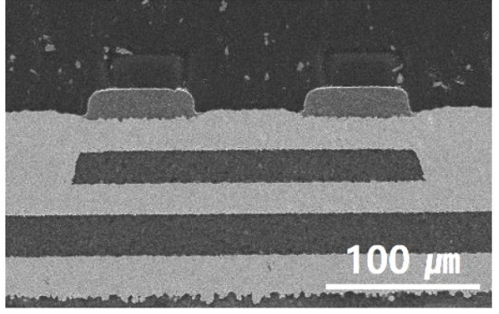

(b)

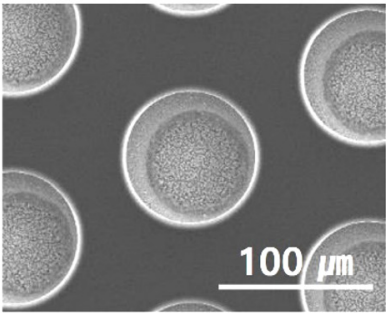

(c)

Fig. 1. Cross-sectional and plane-view images of the PCB pattern used in this experiment (a) A sample was prepared by adding seed sputter deposition and DFR (dry film resistor) coating and patterning on the PCB substrate on which SR (solder resistor) patterning was formed on the final metal wiring. (b) Cross-sectional SEM image, and (c) top view image were shown for the real sample.

씨드층과 리플로우를 통해 만들어지는 금속간 화합물은 용 융되어 다시 응고되는 솔더층의 결정배향에 영향을 주는 것으로 나타나, 이러한 솔더층과 하부 UBM(under bump metallurgy)과의 금속간 화합물에 대한 분석도 필요할 것으 로 판단된다. 따라서, $\mathrm{EBSD}$ 와 $\mathrm{TEM}$ 측정을 통해 도금공 정을 통해 제작된 솔더 범프의 결정학적 미세조직 및 금속 간 화합물의 특징을 분석하고자 한다 [3-6]. 이러한 솔더 도금공정을 통한 접합기술은 이미 $\mathrm{Si}$ 기판상의 $\mathrm{Cu}$ 필라 범프 제작에 활용되어 오고 있으며, 향후 미세화된 $\mathrm{PCB}$ 제조공정에서도 도금공정이 적용될 수 있을 것으로 기대된 다 [7-9].

\section{2. 실험 방법}

$\mathrm{Sn}$ 솔더 범프 제작을 위해 $\mathrm{PCB}$ (printed circuit board) 기판의 metal wiring 상에 기존의 마이크로 볼을 얹기 위 해 형성된 $\mathrm{SR}$ 패턴을 그대로 사용하여 기존 공정과의 호 환성을 높이고자 하였다. 전체 기판에 통전성을 확보하기
위해 구리 씨드층은 스퍼터링을 통해 증착하였다. 이후, SR 패턴만으로는 $\mathrm{Sn}$ 도금시 mushroom 형태로 퍼져서 도금이 진행되기 때문에 패턴 간의 $\mathrm{Sn}$ 도금 부피의 정확한 제어가 어려울 수 있으므로, $\mathrm{SR}$ 패턴 높이보다 두꺼운 $\mathrm{Sn}$ 도금시 에도 비교적 정확한 두께 산포를 확보하기 위해 SR 위에 다시 DFR(dry film resistor)을 코팅하고 이를 다시 패터닝 하였다. 그림 1 에서는 솔더 도금공정 전의 최종 $\mathrm{PCB}$ 패턴 상태의 단면분석 사진 및 모식도를 나타내었다.

그림 2는 전체 공정 모식도를 나타낸 것으로 종래 $\mathrm{SR}$ 패터닝된 기판상에 구리 씨드층 증착 및 $\mathrm{DFR}$ 패터닝이 추 가되며, 이후 $\mathrm{Sn}$ 도금후에는 $\mathrm{DFR}$ stripping 및 불필요한 $\mathrm{Cu}$ 씨드층 제거를 위한 에칭 공정이 추가됨을 알 수 있다. 도금에 필요한 $\mathrm{PCB}$ 기판은 삼성전기에서 제작하였으며, 도금액은 $\mathrm{MSA}$ (methane sulfonic acid)에 기반한 상용 제 품을 사용하였다. $\mathrm{Sn}$ 도금은 전류밀도 $1 \mathrm{ASD}\left(\mathrm{A} / \mathrm{dm}^{2}\right)$ 로 75 분간 진행하였으며, $\mathrm{DFR}$ 제거 후 $240{ }^{\circ} \mathrm{C}, 90 \mathrm{sec}$ 간 수 소분위기하에서 리플로우 열처리를 진행하였다. 표면 및 단 면 형상 관찰을 위해 $\mathrm{FESEM(field} \mathrm{emission} \mathrm{scanning}$

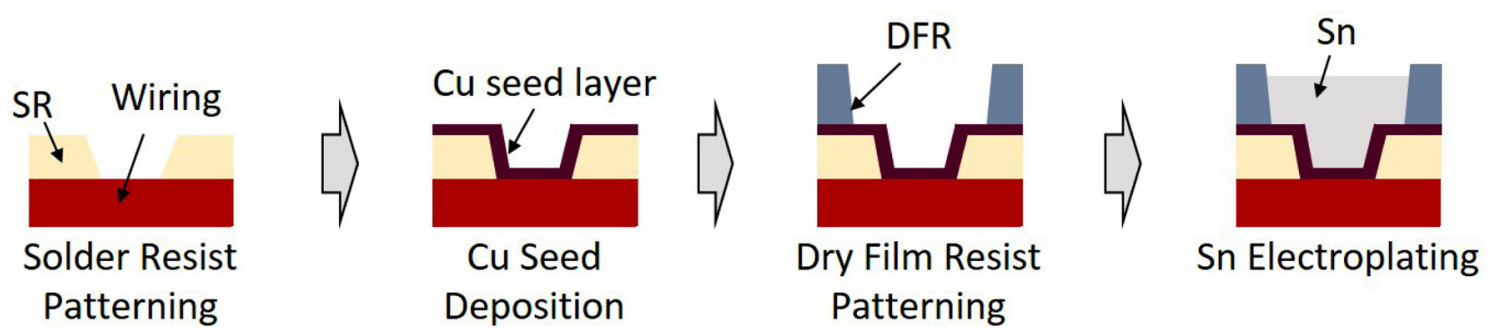

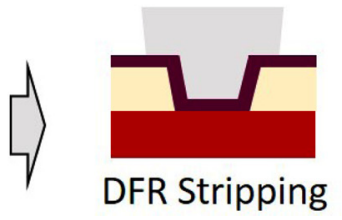

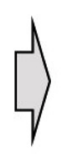

Fig. 2. Schematic diagram of the overall process. DFR patterning is performed on the SR pattern on which the copper seed layer is deposited, and the patterns are filled through a Sn electroplating. After DFR stripping, the bump shape is formed through reflow treatment. 
electron microscopy, FEI Inspect F50)을 사용하였으며, 미세조직 분석을 위한 $\mathrm{EBSD}$ (electron backscattered diffraction) 측정을 위해 $\mathrm{EDAX}$ 사의 Hikari XP EBSD 카메라가 장착된 $\mathrm{FESEM}$ 장치를 이용하여 $\mathrm{EBSD}$ 미세조 직 분석을 진행하였다 [9,10]. 고분해능 미세조직 분석을 위해 Thermo Fisher Scientific사의 FE-STEM(field emission scanning transmission electron microscopy, Talos F200X)를 사용하였으며, 동사의 FIB (focused ion beam, Scios2) 장비를 통해 TEM 시료준비를 진행하였다.

\section{3. 결과 및 고찰}

삼성전기에서 제작한 $\mathrm{PCB}$ 패턴상에 동아대에서 제작한 도금장치를 이용하여 $\mathrm{Sn}$ 도금 시료를 제작하였으며, 도금 시간에 따라 차오르는 형상을 비교하고, 리플로우 후의 형 상을 관찰하였다. 그림 3 은 도금시간 $15,30,45,75$ 분의 4단계로 도금한 결과를 각각 나타내었다. 비교적 conformal 하게 차오르는 것을 알 수 있으며, 리플로우 처리 후에 15 분 도금한 시료에서는 아래로 꺼져 오목한 것이 관찰되 며 $\mathrm{Sn}$ 도금량이 증가함에 따라 위로 볼록해짐을 알 수 있 다. 이전의 $\mathrm{Cu}$ 필라 범프에서 상부의 솔더 영역이 하부의
$\mathrm{Cu}$ 필라의 직경에 의존하여 부분구 형태의 truncated sphere형상을 갖는 것과 마찬가지로, $\mathrm{PCB}$ 솔더 범프에서 도 $\mathrm{SR}$ 패턴상에 증착된 $\mathrm{Cu}$ 씨드층의 영역을 따라 리플로 우 후의 $\mathrm{Sn}$ 이 부분구 형상을 띄는 것으로 나타나며, 그림 3 (a)에서의 15 분 도금한 경우에서는 $\mathrm{Cu}$ 씨드층의 존재 영 역에 비해 $\mathrm{Sn}$ 도금층의 부피가 적은 경우에는 아래로 오 목하게 되는 것을 알 수 있다 $[9,11,12]$. 이 경우에는 truncated sphere형상에서 다소 벗어나는 것을 확인하였다. 그림 4는 $1 \mathrm{ASD}$ 전류밀도 조건에서 75 분간 도금한 시료 에 대해 리플로우 열처리 전후의 단면 $\mathrm{EBSD}$ 미세조직 분 석한 결과를 나타내었다. 리플로우를 통해서 $\mathrm{Sn}$ 층의 미세 조직이 보다 미세해진 것을 알 수 있으며, 특히 $\mathrm{Sn} / \mathrm{Cu}$ 계 면에 IMC (intermetallic compound) 층이 형성된 것을 알 수 있었으며, 대부분 $\mathrm{Cu}_{6} \mathrm{Sn}_{5}$ 결정구조를 나타낸 것으로 상분석을 통해 확인되었다. $\mathrm{EBSD}$ 측정결과는 $\mathrm{Cu}, \mathrm{Sn}$, $\mathrm{Cu}_{6} \mathrm{Sn}_{5}$ 에 대해 각 상의 IPF (inverse pole figure)를 나타 내었으며, 가장 오른쪽 그림에는 각 상의 분포를 색으로 표시하였다. 실제 $\mathrm{Cu}-\mathrm{Sn}$ 계면에 형성되는 $\mathrm{IMC}$ 층은 보통 $\mathrm{Cu}_{6} \mathrm{Sn}_{5}$ 가 scallop형태로 형성되며 $\mathrm{Cu}$ 와 가까운 부분에 $\mathrm{Cu}_{3} \mathrm{Sn}$ 층이 layer 형태로 나타나는 것으로 알려져 있다. 하 지만 $\mathrm{Cu}_{3} \mathrm{Sn}$ 층은 $\mathrm{EBSD}$ 측정에서는 잘 관찰이 되지 않았
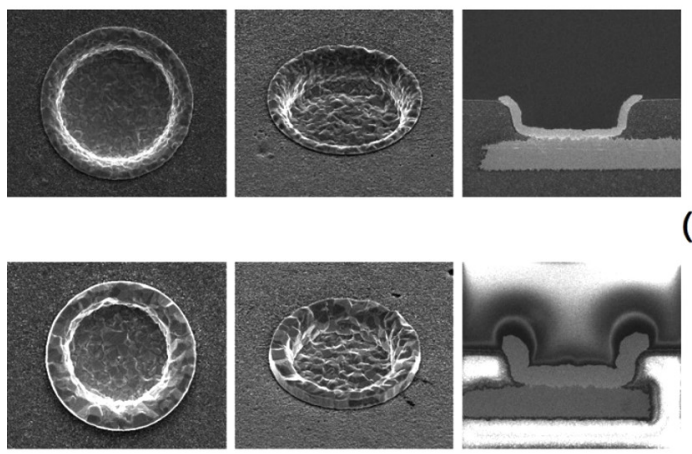

(a)
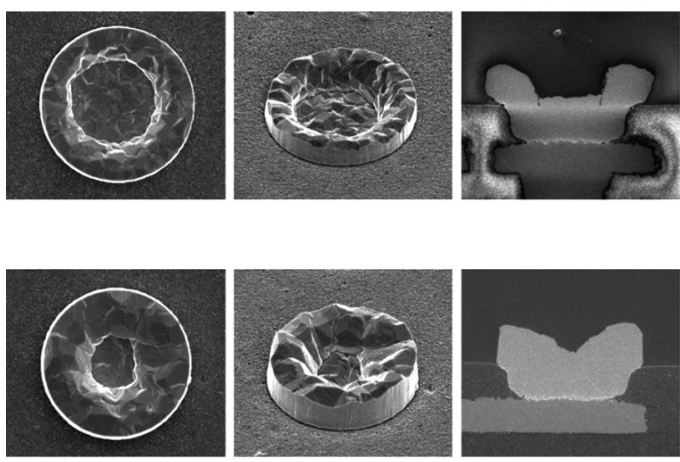

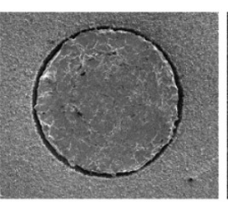

(b)

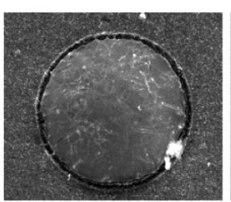

(c)
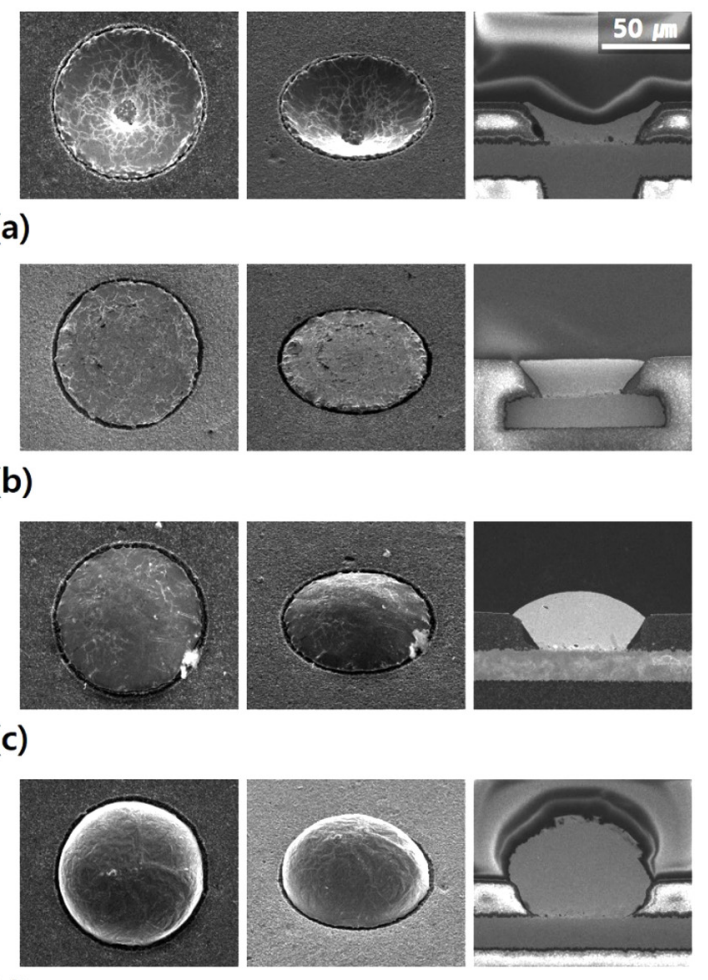

(d)

Fig. 3. Plane-view, tilt and cross-section images of each bump immediately after electroplating and after reflow treatment according to plating time under 1 ASD using MSA-based commercial plating solution. (a) $15 \mathrm{~min}$, (b) $30 \mathrm{~min}$, (c) $45 \mathrm{~min}$, and (d) $75 \mathrm{~min}$. 

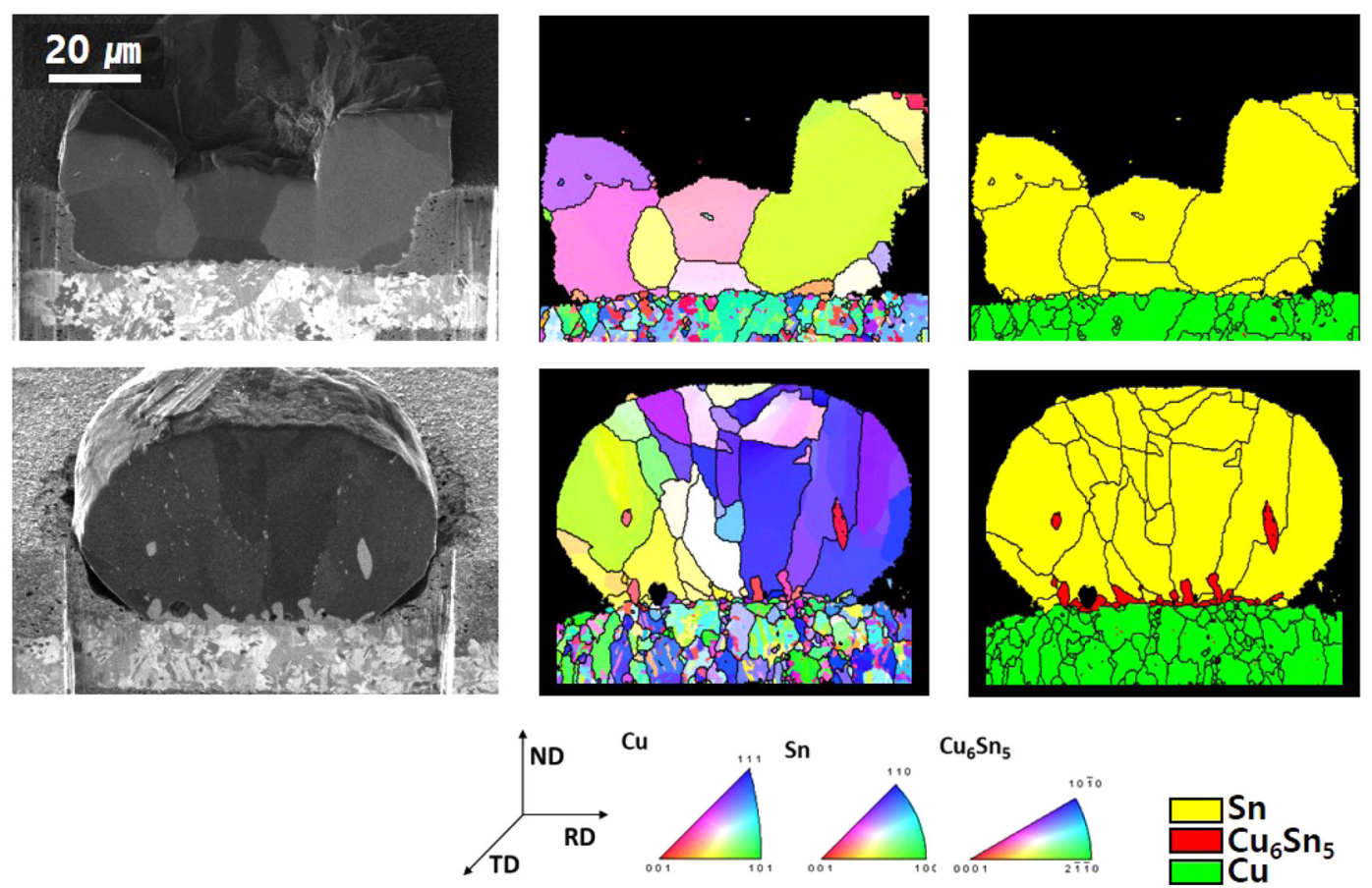

$\mathrm{Cu}_{6} \mathrm{Sn}_{5}$
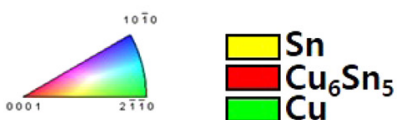

Fig. 4. Cross-sectional FIB milling and EBSD measurement results before (top) and after (bottom) reflow treatment for the solder bump sample.
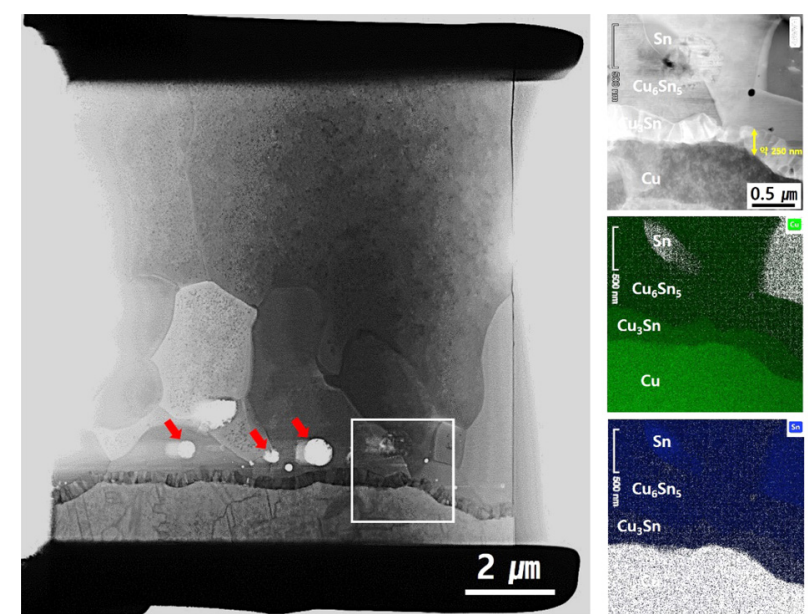

Fig. 5. TEM BF (bright field) image at the $\mathrm{Cu} / \mathrm{Sn}$ interface of a solder bump sample and its HAADF-STEM (high-angle annular dark-field scanning transmission electron microscopy) image and EDS (energy-dispersive X-ray spectroscopy) mapping results of $\mathrm{Cu} / \mathrm{Cu}_{3} \mathrm{Sn} / \mathrm{Cu}_{6} \mathrm{Sn}_{5} / \mathrm{Sn}$ area for a white square area of the BF image. Arrows in red indicate voids formed by vacancy accumulation.

으며, 이를 분석하기 위해 TEM 측정을 진행하였다. 그림 5 에서는 $\mathrm{Cu} / \mathrm{Sn}$ 계면에서의 시료를 채취하여 TEM 명시야 상(bright field image)을 측정한 결과를 나타내고 있으며, 하얀색으로 구획된 정사각형 영역에 대해 HAADF-STEM (high-angle annular dark-field scanning transmission electron microscopy) 이미지와 EDS(energy-dispersive Xray spectroscopy)를 통해 $\mathrm{Cu} / \mathrm{Cu}_{3} \mathrm{Sn}_{2} / \mathrm{Cu}_{6} \mathrm{Sn}_{5} / \mathrm{Sn}$ 영역을 확 인한 결과를 정리하여 나타내었다. 명시야상에서 붉은색 화 살표로 나타낸 바와 같이 상당수의 sub-micron 혹은 나노 스케일의 미세한 void가 형성된 것을 알 수 있는데, 이는 $\mathrm{Sn}$ 도금시에 형성된 vacancy 및 void가 리플로우 과정에 서 agglomeration이 일어나 응집되어 커졌기 때문으로 판 단되며, 확산에 의해 나타나는 계면의 원자 확산계수 차이 에 의해 나타나는 Kirkendall void와는 달리 입내에 형성 된 것이 다수 발견되며 그 경우에 최소 표면적을 갖는 정 원에 가까운 형상을 나타내는 것을 알 수 있다. EDS 분 석 및 HAADF-STEM 분석결과에서 $\mathrm{Cu} / \mathrm{IMC}$ 영역의 차 이를 확실히 확인할 수 있었으며, $\mathrm{Cu}_{3} \mathrm{Sn}$ 층은 layer형태로 서로 연결된 것을 알 수 있다. 반면에 $\mathrm{Cu}_{6} \mathrm{Sn}_{5}$ 가 scallop형 태로 리플로우 공정시에는 액상의 $\mathrm{Sn}$ 내부로 매우 크게 형성된 것을 알 수 있었다. 주목할 점은 $\mathrm{Sn}$ 영역 내부가 뿌옇게 미세한 입자들이 분포한 것을 알 수 있는데, 이에 대해서는 추후에 다시 논의하고자 한다.

그림 6 은 고분해능 $\mathrm{TEM}$ 분석을 진행한 결과로 각각 $\mathrm{Cu} / \mathrm{Cu}_{3} \mathrm{Sn}$ 계면과 $\mathrm{Cu}_{3} \mathrm{Sn}_{2} / \mathrm{Cu}_{6} \mathrm{Sn}_{5}$ 을 관찰한 결과를 나타내었 다. 두 경계면 모두 특정 방향의 관계를 갖기 보다는 곡면 형태의 경계를 갖는 것으로 나타났는데, 이는 $\mathrm{Cu}$ 다결정 구 


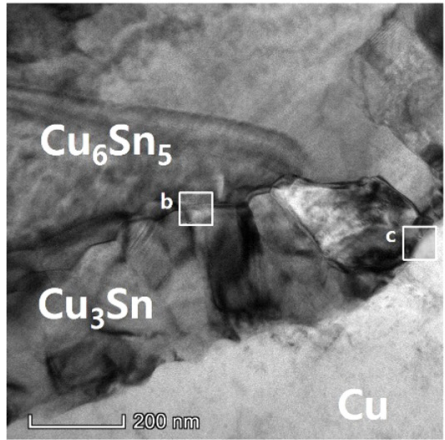

(a)

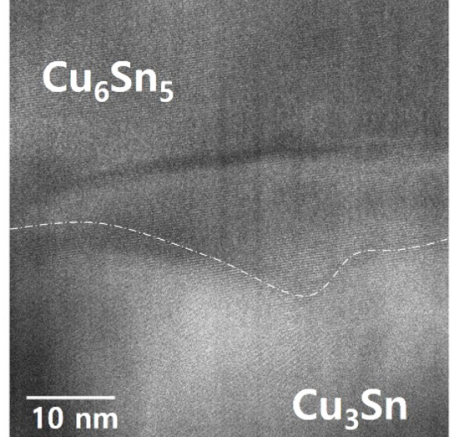

(b)

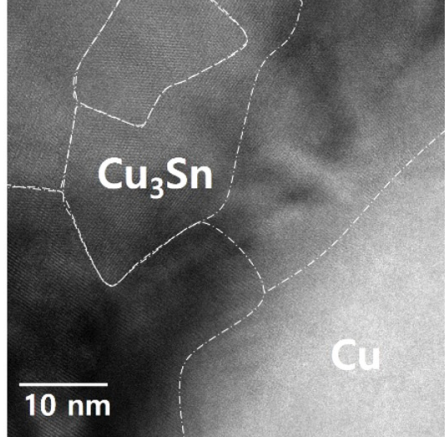

(c)

Fig. 6. High resolution TEM images near $\mathrm{Cu}_{3} \mathrm{Sn} / \mathrm{Cu}_{6} \mathrm{Sn}_{5}$ and $\mathrm{Cu}_{3} \mathrm{Sn} / \mathrm{Cu}$ domain boundaries. (a) High magnification TEM image of a part of the sample in Fig. 5, (b) lattice image of $\mathrm{Cu}_{3} \mathrm{Sn}_{2} \mathrm{Cu}_{6} \mathrm{Sn}_{5}$ boundary, and (c) lattice image of $\mathrm{Cu}_{3} \mathrm{Sn} / \mathrm{Cu}$ boundary.
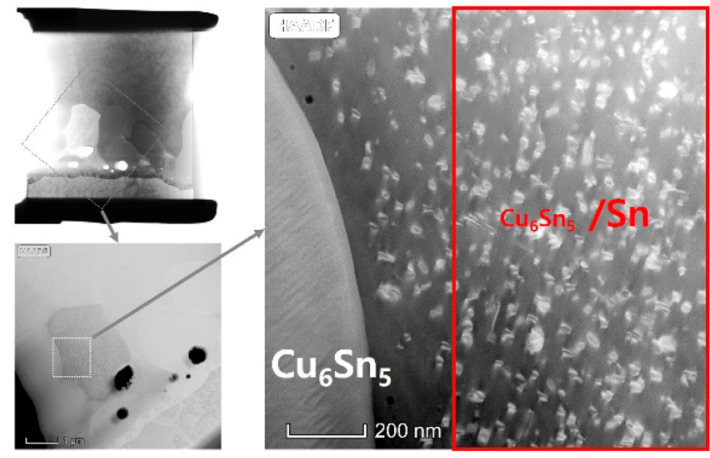

(a)

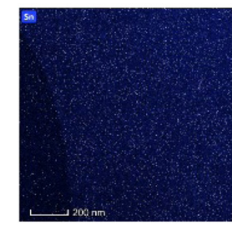

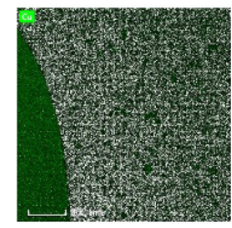

(b)

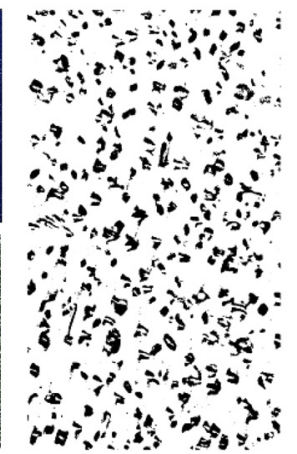

(c)

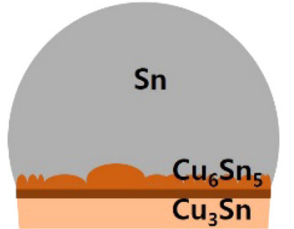

(d)
Fig. 7. TEM and EDS measurement results of $\mathrm{Cu}_{6} \mathrm{Sn}_{5}$ and $\mathrm{Sn}$ phases and its image analysis for calculating the phase fraction. (a) $\mathrm{Cu}_{6} \mathrm{Sn}_{5}$ phase precipitated by eutectic reaction in Sn phase, (b) EDS mapping results, (c) picture showing only $\mathrm{Cu}_{6} \mathrm{Sn}_{5}$ region in the $\mathrm{Sn}$ phase through filtering by image processing using ImageJ software, and (d) schematic diagram of solder bump showing compounds, $\mathrm{Cu}$ and Sn phases.

조(polycrystalline structure)에 기인하는 것으로 생각되며, 추 후 단결정 $\mathrm{Cu}$ 에서의 분석을 진행하면 $\mathrm{Cu}-\mathrm{IMC}$ 및 $\mathrm{IMC}$ 간 의 결정방위의 coherency를 관찰할 수 있을 것으로 기대된
다. $\mathrm{Cu}_{3} \mathrm{Sn}$ 화합물 층의 경우에 수십 $\mathrm{nm}$ 정도의 결정립 크기를 갖는 것으로 나타나 실제 $\mathrm{EBSD}$ 분석이 어려움을 짐작할 수 있었다.

그림 7은 앞서 그림 5 에서 $\mathrm{Sn}$ 영역의 미세한 입자들을 보다 고배율에서 관찰한 결과로, $\mathrm{EDS}$ 분석을 통해 입자가 있는 영역에서 $\mathrm{Cu}$ 농도가 $\mathrm{Cu}_{6} \mathrm{Sn}_{5}$ 화합물의 농도와 거의 비슷한 것을 알 수 있다. 이는 리플로우 공정시 화합물 근 처에서 $\mathrm{Cu}$ 의 용해가 일어나 액상의 $\mathrm{Sn}$ 에 용해되어 들어가 기 때문으로 판단되는데, 열역학적 데이터베이스를 참고하 면 리플로우 공정이 일어나는 온도에서 $\mathrm{Cu}$ 가 약 $1.7 \mathrm{wt} \%$ 의 용해도를 가지는 것으로 확인되었다. 이러한 용해된 $\mathrm{Cu}$ 가 고상으로 되는 공정반응(eutectic reaction)을 통해 고상 의 $\mathrm{Sn}$ 과 $\mathrm{Cu}_{6} \mathrm{Sn}_{5}$ 화합물이 석출되는 경우에 예상되는 부피 분율을 계산하였다. 이 때, $\mathrm{Cu}_{6} \mathrm{Sn}_{5}$ 의 밀도는 $8.26 \mathrm{~g} / \mathrm{cm}^{3}$ 으 로 $\mathrm{Sn}$ 과 $\mathrm{Cu}$ 밀도 $\left(\rho_{\mathrm{Sn}}: 7.27 \mathrm{~g} / \mathrm{cm}^{3}, \rho_{\mathrm{Cu}}: 8.96 \mathrm{~g} / \mathrm{cm}^{3}\right)$ 의 중 간값 정도가 되는 것을 알 수 있는데, $\mathrm{Cu}$ 의 용해도 1.7 $\mathrm{wt} \%$ 에 석출되는 $\mathrm{Cu}_{6} \mathrm{Sn}_{5}$ 의 부피 분율은 약 $4.7 \%$ 로 계산된 다 [13-15]. 하지만, 그림 7(a)의 TEM 측정결과를 바탕으 로 $\mathrm{Cu}_{6} \mathrm{Sn}_{5}$ 의 부피 분율을 측정한 결과에서는 $10 \%$ 정도로 측정되었으며, 이는 예상한 값과 2 배 정도의 큰 차이를 나 타내는데, 이에 대해서는 TEM 이미지상의 번짐 현상으로 인해 정확한 이미지 처리가 어려웠던 점이 있었으며, 그럼 에도 2 배 정도의 큰 차이를 나타낸 것은 실제 용해 공정 의 온도가 높았거나 아니면 평형상이 아닌 과용해 상태에 서 $\mathrm{Cu}$ 의 용해가 진행되었을 가능성이 있을 것으로 추정한 다. 이러한 범프가 놓여지는 하부 UBM (under bump metallurgy)의 구조는 도금방법을 이용하면 수월하게 범프 패턴 내부에 니켈, 코발트, 이리듐 등의 다양한 하부 금속 박막을 전착하는 것이 가능하기 때문에 이러한 박막이 상 부 리플로우 후에 형성되는 $\mathrm{UBM}$ 과 $\mathrm{Sn}$ 사이의 금속간 화 
합물 및 $\mathrm{Sn}$ 의 결정학적 미세구조 형태에 미치는 영향에 대해서는 향후 관련 연구가 추가로 진행될 필요가 있을 것 으로 판단된다 [6].

\section{4. 결 론}

전자부품의 소형화 및 경량화는 실리콘 칩과더불어 이를 탑재하는 $\mathrm{PCB}$ 기판의 미세화를 요구하고 있다. 현재 솔더 범프에 사용되고 있는 마이크로 볼을 $100 \mu \mathrm{m}$ 이하의 미세 패턴에 적용하는데 어려움이 있는 것으로 판단되며, 도금 공정을 통해 이러한 마이크로 볼 공정을 대체하는 방법이 연구되고 있다. 본 연구는 도금공정을 통해 적절한 부피의 $\mathrm{Sn}$ 도금층을 형성하고, 이를 리플로우 공정을 통해 부분구 형상의 솔더 범프형상을 확보하는 것이 가능함을 확인할 수 있었으며, 리플로우 전후 공정에서의 미세조직 변화를 관찰하였다. 특히 리플로우 공정 후의 미세조직에 대해서 고분해능 $\mathrm{TEM}$ 분석을 통해 금속간 화합물과 $\mathrm{Cu}, \mathrm{Sn}$ 간의 경계면 형성에 대한 분석을 진행할 수 있었다. $\mathrm{Cu} / \mathrm{Cu}_{3} \mathrm{Sn}$ 계면의 경우 $\mathrm{EBSD}$ 분석이 어려웠던 이유로 $\mathrm{Cu}_{3} \mathrm{Sn}$ 의 결 정립 크기가 수십 $\mathrm{nm}$ 수준으로 작았기 때문으로 확인되었 으며, $\mathrm{Cu}_{3} \mathrm{Sn} \mathrm{Cu}_{6} \mathrm{Sn}_{5}$ 의 계면은 다결정질로 인해 정확한 계 면 coherency를 관찰하기는 어려웠지만, 완만한 곡면을 가 지고 있음을 확인하였다. 이는 추후 단결정 시료를 통해 보다 확실한 계면분석을 통해 재확인하는 것이 필요할 것 으로 판단되었다. 향후 본 도금공정을 통해 추후 $100 \mu \mathrm{m}$ 이하의 솔더 범프를 $\mathrm{PCB}$ 상에서 안정적으로 제작할 수 있 을 것으로 기대된다.

\section{감사의 글}

이 논문은 교육부와 한국연구재단의 이공학 개인기초연구 지원사업의 지원을 받아 수행된 연구임(NRF-2016R1D1A1B0 2015781).

\section{REFERENCE}

1. Semiconductor Industry Association, International Technology Roadmap for Semiconductors 2007 Edition, https://www. semiconductors.org/resources/2007-international-technologyroadmap-for-semiconductors-itrs/ (2007).

2. K. Toriyama, K. Okamoto, S. Kohara, and Y. Orii, Surface Mount Technol. 12, 49 (2011).

3. Y. Wang, K. H. Lu, V. Gupta, L. Stiborek, D. Shirley, S.-H. Chae, J. Im, and P. S. Ho, J. Mater. Res. 27, 1131 (2012).

4. X. Fu, M. Liu, K. Xu, S. Chen, Y. Shi, Z. Fu, Y. Huang, H. Chen, and R. Yao, Materials 13, 5497 (2020).

5. M. Lu, D.-Y. Shih, P. Lauro, C. Goldsmith, and D. W. Henderson, Appl. Phys. Lett. 92, 211909 (2008).

6. Z. L. Ma, S. A. Belyakov, K. Sweatman, T. Nishimura, and C. M. Gourlay, Nat. Comm. 8, 1916 (2017).

7. T.-G. Woo and I.-S. Park, Korean. J. Met. Mater. 58, 41 (2020).

8. C. Park, B. Yoo, and J. Lee, Korean. J. Met. Mater. 56, 645 (2018).

9. S.-H. Kim, H.-K. Shin, C.-M. Park, D.-U. Kim, P.-R. Cha, U.-H. Lee, and H.-J. Lee, Korean. J. Met. Mater. 53, 495 (2015).

10. S.-H. Kim, H.-J. Lee, D. Josell, and T. P. Moffat, Electrochim. Acta 335, 135612 (2020).

11. K.-N. Chiang and C.-A. Yuan, IEEE Trans. Adv. Packag. 24, 158 (2001).

12. M. J. Pfeifer, IEEE Trans. Comp. Packag. Manufact. Technol. Part B 20, 452 (1997).

13. W.-L. Chen, C.-Y. Yu, C.-Y. Ho, J.-G. Duh, Mat. Sci. Eng. A 613, 193 (2014).

14. J. Feng, C. Hang, Y. Tian, B. Liu, and C. Wang, Sci. Rep. 8, 1775 (2018).

15. S. Shang, A. Kunwar, J. Yao, Y. Wang, H. Ma, and Y. Wang, Met. Mater. Int. 25, 499 (2019). 